\title{
Chebyshev Polynomial Based Numerical Inverse Laplace Transform Solutions of Linear Volterra Integral and Integro-Differential Equations
}

\author{
Vinod Mishra ${ }^{1}$, Dimple Rani \\ Department of Mathematics, Sant Longowal Institute of Engg. \& Tech, Longowal (Punjab)
}

\begin{abstract}
There are enormous occasions when the methods for finding solutions of integral and integrodifferential equations lead to failure because of difficulty in inverting Laplace transform by standard technique. Numerically inverting Laplace transform is cost effective in comparison to rather complicated technique of complex analysis. In the process of numerical inversion, an odd cosine series which is ultimately based on Chebyshev polynomial has been used. The adequacy of method is illustrated through numerical examples of convolution type linear Volterra integral equations of second kind which include weakly singular Abel's integral equation and Volterra integro-differential equation.
\end{abstract}

Keywords: Volterra integral equation; Volterra Integro-differential equation; Numerical Inversion of Laplace transform; Gaussian quadrature; Cosine series; Chebyshev polynomial.

\section{INTRODUCTION}

Linear Volterra Integral and Integro-Differential equations are extensively used in the various specialties of science and engineering. These include mathematical physics, chemical kinetic, heat conduction, seismology, fluid dynamics, biological models, population dynamics, metallurgy and semi-conductors [11, $12 \& 13]$.

The Volterra Integral equation with a convolution kernel is defined by

$$
f(x)=y(x)+\int_{0}^{x} k(x-t) f(t) d t, \quad x \in[0, T]
$$

while Volterra Integro-Differential equation by

$$
u^{\prime}(x)=\gamma+\alpha u(x)+\beta \int_{x_{0}}^{x} k(x-t) u(x) d x, u\left(x_{0}\right)=u_{0},
$$

There have been many extant methods for solving Volterra Integral and Integro-Differential equations with convolution kernels. Wazwaz[12] and Al-Hayani[18] discussed the Adomian polynomial based Laplace Transform methods to solve these equations. Yang[13] proposed a method by which solution is expressed in power series and to improve the convergence rate applied the Pade approximant. Babolian-Shamloo[7], Aznam-Hussin[17] and Mishra et al.[9] used operational matrices of piecewise constant orthogonal function or Haar wavelet to solve these equations. Homotopy perturbation method with finite difference technique was used by Raftari[10] to solve Volterra Integro-Differential equations. Zarebnia[14] solved these equations using Sinc function. A modified Taylor series method has been applied to approximate the solution of linear Integro-Differential equations in [11].

\subsection{Numerical Inverse Laplace Transform}

The Laplace transform of function is defined by

$$
L[f(t)]=F(s)=\int_{0}^{\infty} e^{-s t} f(t) d t .
$$

\footnotetext{
${ }^{1}$ Corresponding Author: vinodmishra.2011@ rediffmail.com
} 


\section{American Research Journal of Mathematics, Volume 1, Issue 1, February 2015}

ISSN 2378-704X

As usual, the equations are first converted into algebraic equations using Laplace transform. The inverse Laplace transform is then applied and the numerical solution is finally expressed in terms of Chebyshev series.

Applying Laplace transforms on both sides of above equations and then using convolution property in (1) \& (2), reduce the equations in the form

$$
L[f(x)]=F(s) \text {. }
$$

The inversion leads to

$$
f(x)=L^{-1} F(s) .
$$

Comprehensive literature consists of a number of methods for numerically inverting Laplace transform suited for problems in particular situations. For a detailed survey of various methods for Laplace transform inversion numerically refers to Cohen[2], Davies-Martin[5] and Mishra[16]. Bellman et al. (1966)[1] have outlined a method which they derive from the consideration of Gauss-Legendre's quadrature rule. Substituting $x=e^{-\sigma t}, \sigma>0$ in [1 \& 2], $F(s)$ is transformed from the interval $(0, \infty)$ to $(0,1)$ as

$F(s)=\frac{1}{\sigma} \int_{0}^{1} x^{s / \sigma-1} g(x) d x$, Where $g(x)=f\left(-\frac{1}{\sigma}(\log x)\right)$.

$F(s)$ can be numerically inverted using Legendre series of $g(x)=\sum_{k=0}^{\infty} \alpha_{k} P_{2 k}(x)$ by considering $g(x)$ being even function in $[-1,1]$. This method has slow convergence as the coefficients $\alpha_{k}$ decrease slowly due to the singularity of $g(x)$ at $x=0\left[\begin{array}{ll}1 & \&\end{array}\right.$ 2]. Erdelyi (1943), Papoulis (1956) and Lanczos (1957) have proposed Legendre's function to find the approximate value of function $f(t)$ [2, 5, 6, \& 8]. In [3] Dubner and Abate (1968) have expressed the $f(t)$ in terms Fourier cosine transforms. Durbin (1974) [4] has proposed the trapezoidal rule by resulting approximation.

\subsection{Chebyshev Series based Inversion}

Chebyshev polynomial is due to Panfnuty Chebyshev (b. 1821), a Russian. It is basically a class of orthogonal polynomials. In sixties remarkable development to the theory lead to computation of function approximations, integrals and solution of differential equations using Chebyshev polynomials, termed as Chebyshev series expansion of a function. The range of problems covered includes singular problem and network synthesis [15].

Here we propose a technique parallel to Papoulis[6] by making the substitution

$$
\sin \theta=e^{-\sigma x}, \sigma>0 \text {. }
$$

The interval $(0, \infty)$ transformed into $\left(0, \frac{\pi}{2}\right)$ and $f(x)$ becomes

$$
f\left(-\frac{1}{\sigma} \log (\sin \theta)\right)=g(\theta) \text {. }
$$

Now eq. (3) takes the form

$$
\sigma F(s)=\int_{0}^{\frac{\pi}{2}}(\sin \theta)^{\frac{s}{\sigma}-1} \cos \theta g(\theta) d \theta .
$$

By setting $s=(2 k+1) \sigma, k=0,1,2 \ldots$, we have 
$\sigma F((2 k+1) \sigma)=\int_{0}^{\frac{\pi}{2}}(\sin \theta)^{2 k} \cos \theta g(\theta) d \theta$

Here we assume that $g\left(\frac{\pi}{2}\right)=f(0)=0$. In case this does not hold then arrange it by subtracting a suitable function from $g(\theta)$. The function $g(\theta)$ can be expanded in $\left(0, \frac{\pi}{2}\right)$ as the odd cosine series

$g(\theta)=\sum_{k=0}^{\infty} \alpha_{k} \cos (2 k+1) \theta$

and is valid in the interval $\left(-\frac{\pi}{2}, \frac{\pi}{2}\right)$.

Now we have to determine the coefficients $\alpha_{k}$

$(\sin \theta)^{2 k} \cos \theta=\left(\frac{e^{i \theta}-e^{-i \theta}}{2 i}\right)^{2 k}\left(\frac{e^{i \theta}+e^{-i \theta}}{2}\right)$.

Making substitution $x=e^{i \theta}$, we find that

$2^{2 k}(-1)^{k}(\sin \theta)^{2 k} \cos \theta=$

$\frac{1}{2}\left(x-\frac{1}{x}\right)^{2 k}\left(x+\frac{1}{x}\right)=\frac{1}{2}\left[x^{2 k+1}+\left(\frac{1}{x^{2 k+1}}\right)\right]+\frac{1}{2} x^{2 k-1}\left[\left(\begin{array}{l}2 k \\ 0\end{array}\right)-\left(\begin{array}{l}2 k \\ 1\end{array}\right)\right]+\frac{1}{2}\left(\frac{1}{x}\right)^{2 k-1}\left[\left(\begin{array}{l}2 k \\ 2 k\end{array}\right)-\left(\begin{array}{l}2 k \\ 2 k-1\end{array}\right)\right]+\ldots$

$\left[\frac{1}{2} x^{2 k-2 r+1}\left\{(-1)^{r}\left(\begin{array}{l}2 k \\ r\end{array}\right)+(-1)^{r-1}\left(\begin{array}{l}2 k \\ r-1\end{array}\right)\right\}\right]+\left[\frac{1}{2}\left(\frac{1}{x}\right)^{2 k-2 r+1}\left\{(-1)^{2 k-r}\left(\begin{array}{l}2 k \\ 2 k-r\end{array}\right)+(-1)^{2 k-r+1}\left(\begin{array}{l}2 k \\ 2 k-r+1\end{array}\right)\right\}+\ldots\right.$

$\left[\frac{1}{2} x\left\{(-1)^{k}\left(\begin{array}{l}2 k \\ k\end{array}\right)+(-1)^{k-1}\left(\begin{array}{l}2 k \\ k-1\end{array}\right)\right\}\right]+\left[\frac{1}{2}\left(\frac{1}{x}\right)\left\{(-1)^{k}\left(\begin{array}{l}2 k \\ k\end{array}\right)+(-1)^{k+1}\left(\begin{array}{l}2 k \\ k+1\end{array}\right)\right\}\right] \ldots$

$=\cos (2 k+1)+\ldots+(-1)^{r-1}\left[\left(\begin{array}{l}2 k \\ r-1\end{array}\right)-\left(\begin{array}{l}2 k \\ r\end{array}\right)\right] \cos (2 k-2 r+1) \theta \ldots+(-1)^{k-1}\left[\left(\begin{array}{l}2 k \\ k-1\end{array}\right)-\left(\begin{array}{l}2 k \\ k\end{array}\right)\right] \cos \theta$

Substitution of (8) \& (9) and using the result of the orthogonality

$\int_{0}^{\pi / 2}[\cos (2 k+1) \theta]^{2} d \theta=\frac{\pi}{4}$

eq. (7) gives

$\sigma F((2 k+1) \sigma)=$

$\frac{1}{2^{2 k}(-1)^{k}} \frac{\pi}{4}\left\{(-1)^{k-1}\left[\left(\begin{array}{l}2 k \\ k-1\end{array}\right)-\left(\begin{array}{l}2 k \\ k\end{array}\right)\right] \alpha_{0} \ldots+(-1)^{r-1}\left[\left(\begin{array}{l}2 k \\ r-1\end{array}\right)-\left(\begin{array}{l}2 k \\ r\end{array}\right)\right] \alpha_{k-r}+\ldots .+\alpha_{k}\right\}$ 


$$
\begin{aligned}
& \frac{4}{\pi} 2^{2 k} \sigma F((2 k+1) \sigma)= \\
& \frac{1}{(-1)^{k}}\left\{(-1)^{k-1}\left[\left(\begin{array}{l}
2 k \\
k-1
\end{array}\right)-\left(\begin{array}{l}
2 k \\
k
\end{array}\right)\right] \alpha_{0} \ldots+(-1)^{r-1}\left[\left(\begin{array}{l}
2 k \\
r-1
\end{array}\right)-\left(\begin{array}{l}
2 k \\
r
\end{array}\right)\right] \alpha_{k-r}+\ldots+\alpha_{k}\right\}
\end{aligned}
$$

which is the linear system in $\alpha_{0}, \alpha_{1}, \alpha_{2} \ldots . . \alpha_{k} \ldots$. It can be conveniently put in the matrix form

$$
C=A B,
$$

where the matrix $C$ and the coefficient matrix A can be obtained by putting the values of $k=0,1,2, \ldots$ in LHS and RHS of (11) respectively.

$$
A=\left[\begin{array}{ccccc}
1 & 0 & 0 & \cdots & \cdots \\
1 & -1 & 0 & \cdots & \cdots \\
2 & -3 & 1 & \cdots & \cdots \\
\vdots & \vdots & \vdots & \cdots & \cdots \\
-\left[\left(\begin{array}{c}
2 k \\
k-1
\end{array}\right)-\left(\begin{array}{l}
2 k \\
k
\end{array}\right)\right] & \cdots & \cdots & \cdots & (-1)^{-k}
\end{array}\right] \quad, \quad B=\left[\begin{array}{c}
\alpha_{0} \\
\alpha_{1} \\
\alpha_{2} \\
\vdots \\
\alpha_{k}
\end{array}\right]
$$

Thus $\alpha_{k}$ can be obtained by solving $B=A^{-1} C$ and hence $g(\theta)$ can be obtained from eq. (7).

In general, we compute first $(N+1)$ terms of eq.(8), that is, the finite series

$$
g_{N}(\theta)=\sum_{k=0}^{N} \alpha_{k} \cos (2 k+1) \theta
$$

As $N \rightarrow \infty, g_{N}(\theta) \rightarrow g(\theta)$. From $g(\theta)$ we can determine $f(x)$

$f(x)=\sum_{k=0}^{\infty} \alpha_{k} \cos (2 k+1) \theta$.

Defining $U_{k-1}(x)=\frac{\cos k \theta}{\cos \theta}, x=\sin \theta$, where $U_{k}(x)$ is the Chebyshev polynomial of second kind of degree $k$ Then

$$
\cos \theta=\left(1-e^{-2 \sigma x}\right)^{1 / 2}
$$

And

$$
f(x)=\left(1-e^{-2 \sigma x}\right)^{1 / 2} \sum_{k=0}^{\infty} \alpha_{k} U_{2 k}\left(e^{-\sigma x}\right) .
$$

\subsection{Numerical Examples}

\section{Example1}

Consider the weakly singular Volterra integral equation of second kind [12\&13] 
American Research Journal of Mathematics, Volume 1, Issue 1, February 2015

ISSN 2378-704X

$$
u(x)=2 \sqrt{x}-\int_{0}^{x} \frac{u(t)}{\sqrt{x-t}} d t, x \in[0,1]
$$

Taking Laplace transform on both sides of eq. (15) and using convolution theorem, we obtain

$$
L(u)=\frac{\sqrt{\pi}}{s(\sqrt{s}+\sqrt{\pi})}=F(s) .
$$

The necessary condition for compliance of inverse $u(x)$ of eq.(16) is that $u(0)=0$. Following initial value theorem,

$$
u(0)=\lim _{s \rightarrow \infty} s F(s)=\lim _{s \rightarrow \infty} s \frac{\sqrt{\pi}}{s(\sqrt{s}+\sqrt{\pi})}=0 .
$$

Therefore, the solution $u(x)$ can be obtained and $\alpha_{k}$ can be computed using relation (12).

Table1.1. Coefficients in the Expansion of $u(x)$

\begin{tabular}{|c|c|}
\hline $\mathrm{k}$ & $\alpha_{k}$ \\
\hline 0 & 0.81399311 \\
\hline 1 & -0.0446193 \\
\hline 2 & 0.03972445 \\
\hline 3 & 0.00011582 \\
\hline 4 & 0.01165101 \\
\hline 5 & 0.00230399 \\
\hline 6 & 0.00569674 \\
\hline 8 & 0.00212046 \\
\hline 9 & 0.00350064 \\
\hline 10 & 0.00166594 \\
\hline & 0.00164977 \\
\hline
\end{tabular}

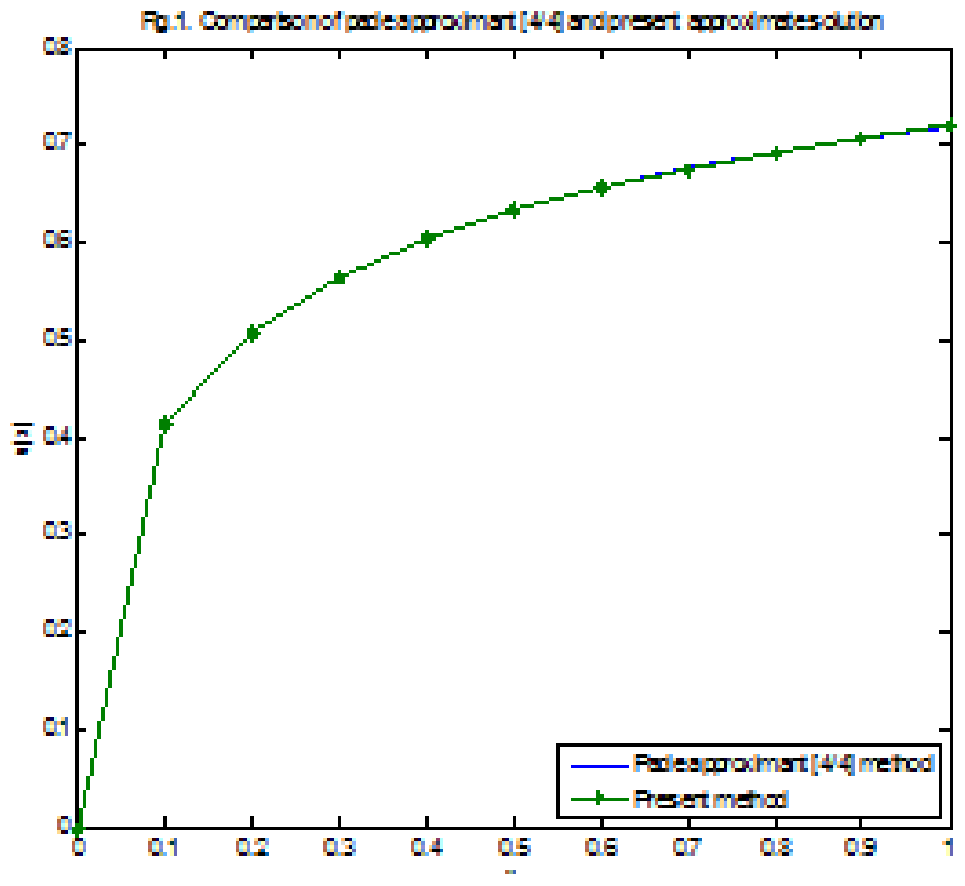


American Research Journal of Mathematics, Volume 1, Issue 1, February 2015

ISSN 2378-704X

Table1.2. Computed Pade approximant [4/4] and Approximation Solution for Example1

\begin{tabular}{|c|c|c|c|}
\hline$X$ & Pade approximant [4/4] method & Present method & Absolute Error \\
\hline 0 & 0 & $5.14 \mathrm{E}-17$ & $5.13800 \mathrm{E}-17$ \\
\hline 0.1 & 0.41411018 & 0.4141034 & $6.78000 \mathrm{E}-06$ \\
\hline 0.2 & 0.50848304 & 0.5087519 & $2.68860 \mathrm{E}-04$ \\
\hline 0.3 & 0.56452274 & 0.5637984 & $7.24340 \mathrm{E}-04$ \\
\hline 0.4 & 0.60364034 & 0.6033569 & $2.83440 \mathrm{E}-04$ \\
\hline 0.5 & 0.63323515 & 0.6338924 & $6.57250 \mathrm{E}-04$ \\
\hline 0.6 & 0.65675942 & 0.655994 & $7.65420 \mathrm{E}-04$ \\
\hline 0.7 & 0.67610075 & 0.6740862 & $2.01455 \mathrm{E}-03$ \\
\hline 0.8 & 0.69240064 & 0.6911992 & $1.20144 \mathrm{E}-03$ \\
\hline 0.9 & 0.70639982 & 0.7070156 & $6.15780 \mathrm{E}-04$ \\
\hline 1 & 0.71860476 & 0.7202293 & $1.62454 \mathrm{E}-03$ \\
\hline
\end{tabular}

Example2. Consider the integral equation

$$
\int_{0}^{x} u(t) u(x-t) d t=\sin x .
$$

Laplace transform on both sides of eq. (17) gives

$L(u)=\frac{1}{\sqrt{s^{2}+1}}=F(s)$.

The condition $u(0)=0$ is not satisfied

$$
u(0)=\lim _{s \rightarrow \infty} s F(s)=\lim _{s \rightarrow \infty} s \frac{1}{\sqrt{s^{2}+1}}=1 .
$$

As per provision of condition a possible function need to be subtracted from $u(x)$ is 1 . A function which takes the value 1 at $x=0$ is $e^{-x}$. Therefore, $U(x)=u(x)-e^{-x}$ as $U(0)=0$. Since $\bar{F}(s)=F(s)-\frac{1}{s+1}$. Thereby, the solution $u(x)$ can be obtained by computing the coefficients $\alpha_{k}$ the relation (12).

Table2.1. Coefficients in the Expansion of $u(x)$

\begin{tabular}{|c|c|}
\hline $\mathrm{k}$ & $\alpha_{k}$ \\
\hline 0 & 0.26369654 \\
\hline 1 & -0.0735987 \\
\hline 2 & -0.1482495 \\
\hline 3 & -0.0985091 \\
\hline 4 & -0.0769397 \\
\hline 5 & -0.0547004 \\
\hline 6 & -0.042268 \\
\hline 7 & -0.0310263 \\
\hline 8 & -0.0239681 \\
\hline 10 & -0.0176349 \\
\hline 11 & -0.0133825 \\
\hline 12 & -0.0095073 \\
\hline 13 & -0.0067188 \\
\hline 14 & -0.003956 \\
\hline
\end{tabular}


American Research Journal of Mathematics, Volume 1, Issue 1, February 2015

ISSN 2378-704X

Table2.2. Computed Exact and Approximation Solution for Example2

\begin{tabular}{|c|c|c|c|}
\hline $\mathrm{x}$ & Exact solution & Present method & Absolute error \\
\hline 0 & 1 & 1 & $3.00000 \mathrm{E}-09$ \\
\hline 0.1 & 0.99750157 & 0.99722919 & $2.72375 \mathrm{E}-04$ \\
\hline 0.2 & 0.99002498 & 0.98950321 & $5.21765 \mathrm{E}-04$ \\
\hline 0.3 & 0.97762625 & 0.97849647 & $8.70222 \mathrm{E}-04$ \\
\hline 0.4 & 0.96039823 & 0.95925921 & $1.13902 \mathrm{E}-03$ \\
\hline 0.5 & 0.93846981 & 0.93944053 & $9.70723 \mathrm{E}-04$ \\
\hline 0.6 & 0.91200486 & 0.9125871 & $5.82237 \mathrm{E}-04$ \\
\hline 0.7 & 0.88120089 & 0.87896932 & $2.23157 \mathrm{E}-03$ \\
\hline 0.8 & 0.84628735 & 0.84616791 & $1.19441 \mathrm{E}-04$ \\
\hline 0.9 & 0.8075238 & 0.81065911 & $3.13531 \mathrm{E}-03$ \\
\hline 1 & 0.76519768 & 0.7670378 & $1.84012 \mathrm{E}-03$ \\
\hline
\end{tabular}

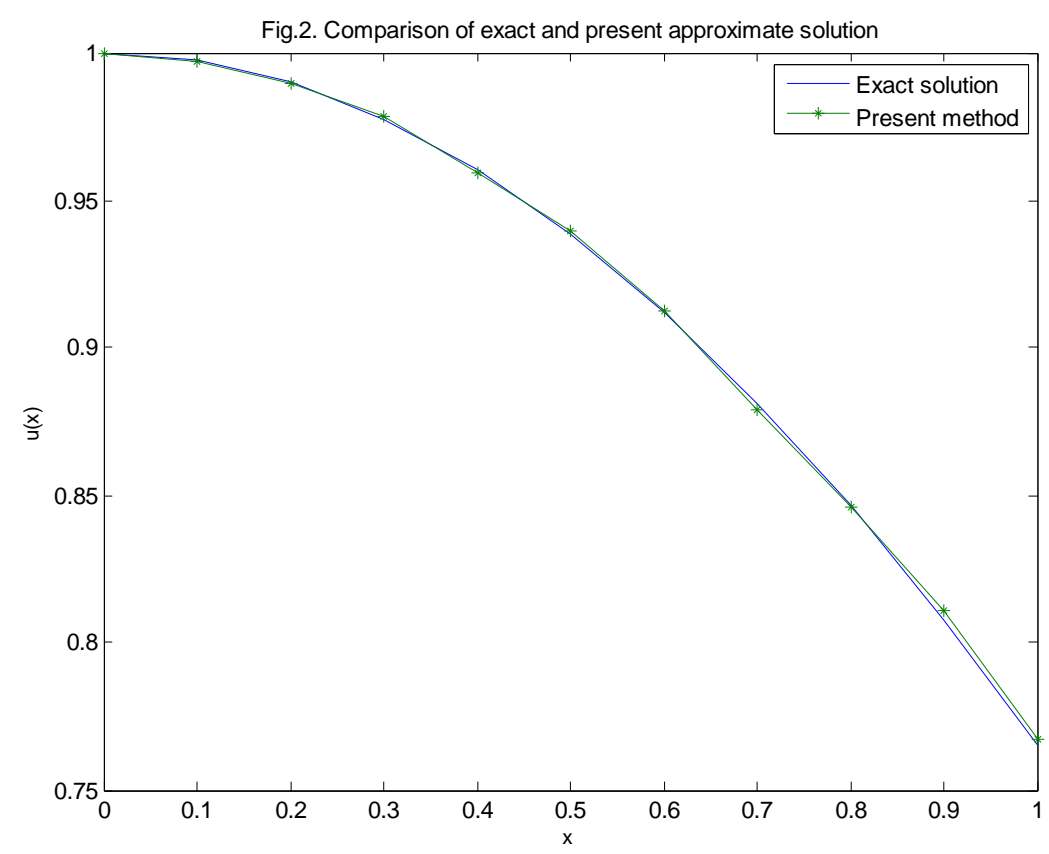

Example3. Consider the Volterra integral with a convolution kernel given by [13]

$$
u(x)+\int_{0}^{x} \cos (x-t) u(t) d t=\sin x
$$

As usual taking Laplace transform on both sides of eq.(19) yield

$$
L(u)=\frac{1}{s^{2}+s+1}=F(s) .
$$

The condition $u(0)=0$ is satisfied as in Example 1, that is

$u(0)=\lim _{s \rightarrow \infty} s F(s)=\lim _{s \rightarrow \infty} s \frac{1}{s^{2}+s+1}=0$. 
American Research Journal of Mathematics, Volume 1, Issue 1, February 2015

ISSN 2378-704X

Therefore, the solution $u(x)$ is feasible. The coefficients $\alpha_{k}$ computed (12) are shown below.

Table3.1.Coefficients in the Expansion of $u(x)$

\begin{tabular}{|c|c|}
\hline $\mathrm{k}$ & $\alpha_{k}$ \\
\hline 0 & 0.42441318 \\
\hline 1 & 0.03264717 \\
\hline 2 & -0.09372896 \\
\hline 3 & -0.07000573 \\
\hline 4 & -0.06126397 \\
\hline 5 & -0.04672609 \\
\hline 6 & -0.0391798 \\
\hline 7 & -0.03125062 \\
\hline 8 & -0.02649286 \\
\hline 9 & -0.02180741 \\
\hline 10 & -0.01875585 \\
\hline 11 & -0.01580044 \\
\hline 12 & -0.01381433 \\
\hline 13 & -0.01199908 \\
\hline 14 & -0.01103245 \\
\hline
\end{tabular}

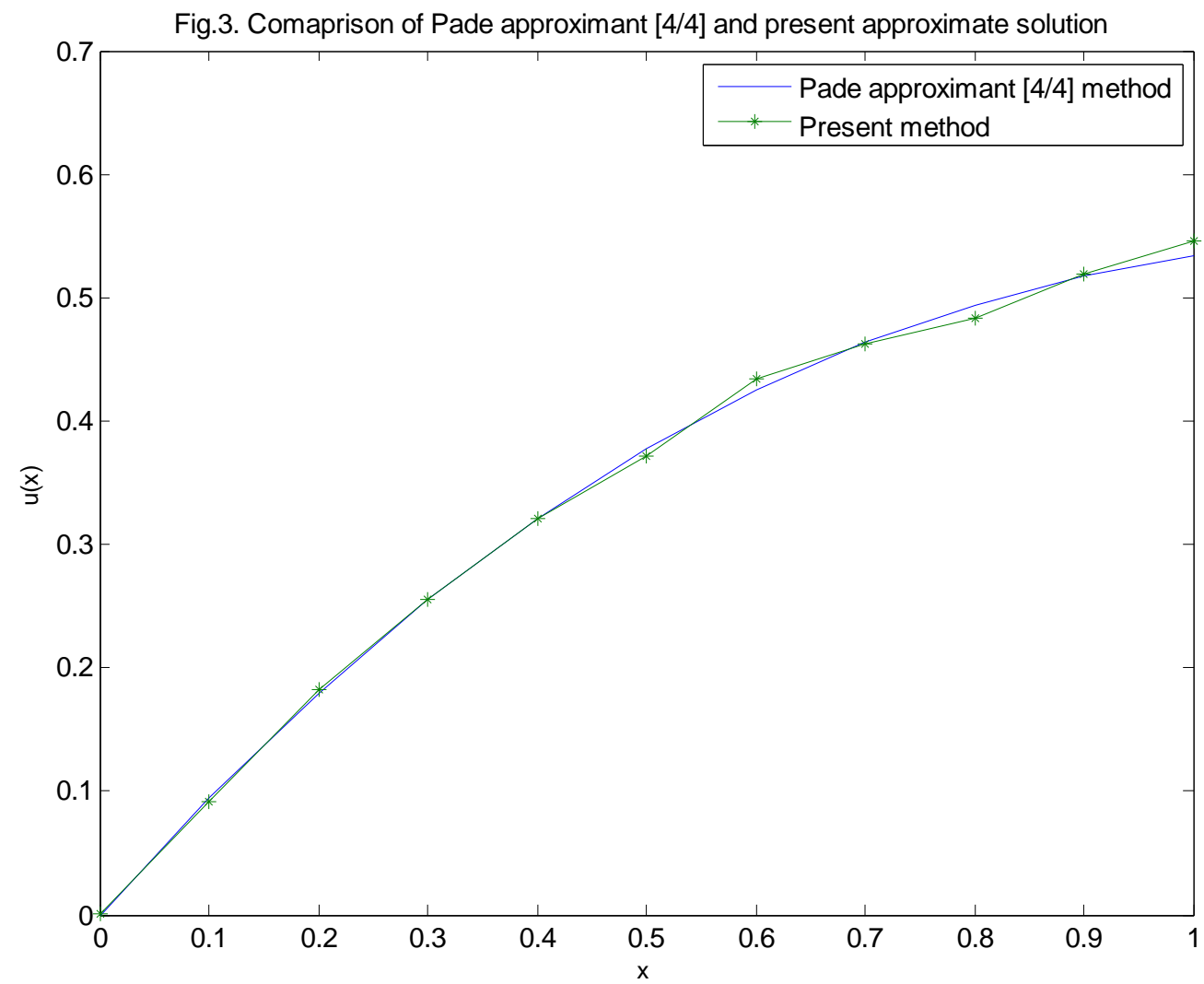


American Research Journal of Mathematics, Volume 1, Issue 1, February 2015

ISSN 2378-704X

Table 3.2. Computed Pade approximant [4/4] and Approximation Solution for Example3

\begin{tabular}{|c|c|c|c|}
\hline$X$ & Pade approximant [4/4] & Present method & Absolute error \\
\hline 0 & 0 & $4.12 \mathrm{E}-16$ & $3.12297 \mathrm{E}-16$ \\
\hline 0.1 & 0.095004082 & 0.09158784 & $3.03423 \mathrm{E}-03$ \\
\hline 0.2 & 0.180063957 & 0.183098185 & $8.34922 \mathrm{E}-04$ \\
\hline 0.3 & 0.255316973 & 0.256151895 & $1.98599 \mathrm{E}-04$ \\
\hline 0.4 & 0.320980415 & 0.321179014 & $5.24466 \mathrm{E}-03$ \\
\hline 0.5 & 0.377341791 & 0.372097134 & $9.20657 \mathrm{E}-03$ \\
\hline 0.6 & 0.42474943 & 0.433955997 & $1.71357 \mathrm{E}-03$ \\
\hline 0.7 & 0.463603433 & 0.461889867 & $1.07646 \mathrm{E}-02$ \\
\hline 0.8 & 0.494347029 & 0.483582381 & $1.12133 \mathrm{E}-03$ \\
\hline 0.9 & 0.517458374 & 0.518579699 & $1.30089 \mathrm{E}-02$ \\
\hline 1 & 0.533442822 & 0.546451691 & \\
\hline
\end{tabular}

Example4. Consider the first order linear Volterra Integro-differential equation of the form [8]

$u^{\prime}(x)=\gamma+\alpha u(x)+\beta \int_{x_{0}}^{x} e^{-\delta(x-t)} u(t) d t, u\left(x_{0}\right)=u_{0}$.

If we choose $\gamma=0, \alpha=0, \beta=-1, \delta=1, x_{0}=0, u_{0}=1$.

Taking Laplace transform on both sides of eq. (21) and using derivative property and convolution theorem of Laplace transform,

$L(u)=\frac{s+1}{s^{2}+s+1}=F(s)$.

The condition $u(0)=0$ is not satisfied as

$u(0)=\lim _{s \rightarrow \infty} s F(s)=\lim _{s \rightarrow \infty} s \frac{s+1}{s^{2}+s+1}=1$.

In this case we choose a possible function that will be subtracted from $u(x)$ to be 1 . A function which takes the value 1 at $x=0$ is $e^{-x}$. Therefore, $U(x)=u(x)-e^{-x}$ as $U(0)=0$. Since $\bar{F}(s)=F(s)-\frac{1}{s+1}$, the solution $u(x)$ exists. The coefficients $\alpha_{k}$ can be calculated using the relation (12).

Table 4.1. Coefficients in the Expansion of $u(x)$

\begin{tabular}{|c|c|}
\hline $\mathrm{k}$ & $\alpha_{k}$ \\
\hline 0 & 0.212206589 \\
\hline 1 & -0.08161792 \\
\hline 2 & -0.12163703 \\
\hline 3 & -0.06349292 \\
\hline 4 & -0.04422927 \\
\hline 5 & -0.02637033 \\
\hline 6 & -0.01850358 \\
\hline 8 & -0.01134116 \\
\hline 9 & -0.00779918 \\
\hline 10 & -0.00443629 \\
\hline 11 & -0.00269506 \\
\hline & -0.00095958 \\
\hline
\end{tabular}


American Research Journal of Mathematics, Volume 1, Issue 1, February 2015

ISSN 2378-704X

12

$-5.86 \mathrm{E}-05$

Table4.2. Computed Exact and Approximation Solution for Example4

\begin{tabular}{|c|c|c|c|}
\hline $\mathrm{x}$ & Exact solution & Present method & Absolute error \\
\hline 0 & 1 & 1 & $0.00 \mathrm{E}+00$ \\
\hline 0.1 & 0.9951666 & 0.99489203 & $2.75 \mathrm{E}-04$ \\
\hline 0.2 & 0.9813308 & 0.98137139 & $4.06 \mathrm{E}-05$ \\
\hline 0.3 & 0.9594808 & 0.95941456 & $6.62 \mathrm{E}-05$ \\
\hline 0.4 & 0.930587 & 0.93094843 & $3.61 \mathrm{E}-04$ \\
\hline 0.5 & 0.8955945 & 0.89502027 & $5.74 \mathrm{E}-04$ \\
\hline 0.6 & 0.8554164 & 0.85534569 & $7.07 \mathrm{E}-05$ \\
\hline 0.7 & 0.8109282 & 0.81181337 & $8.85 \mathrm{E}-04$ \\
\hline 0.8 & 0.762963 & 0.76330526 & $3.42 \mathrm{E}-04$ \\
\hline 0.9 & 0.7123077 & 0.71129202 & $1.02 \mathrm{E}-03$ \\
\hline 1 & 0.6597002 & 0.65824129 & $1.46 \mathrm{E}-03$ \\
\hline
\end{tabular}

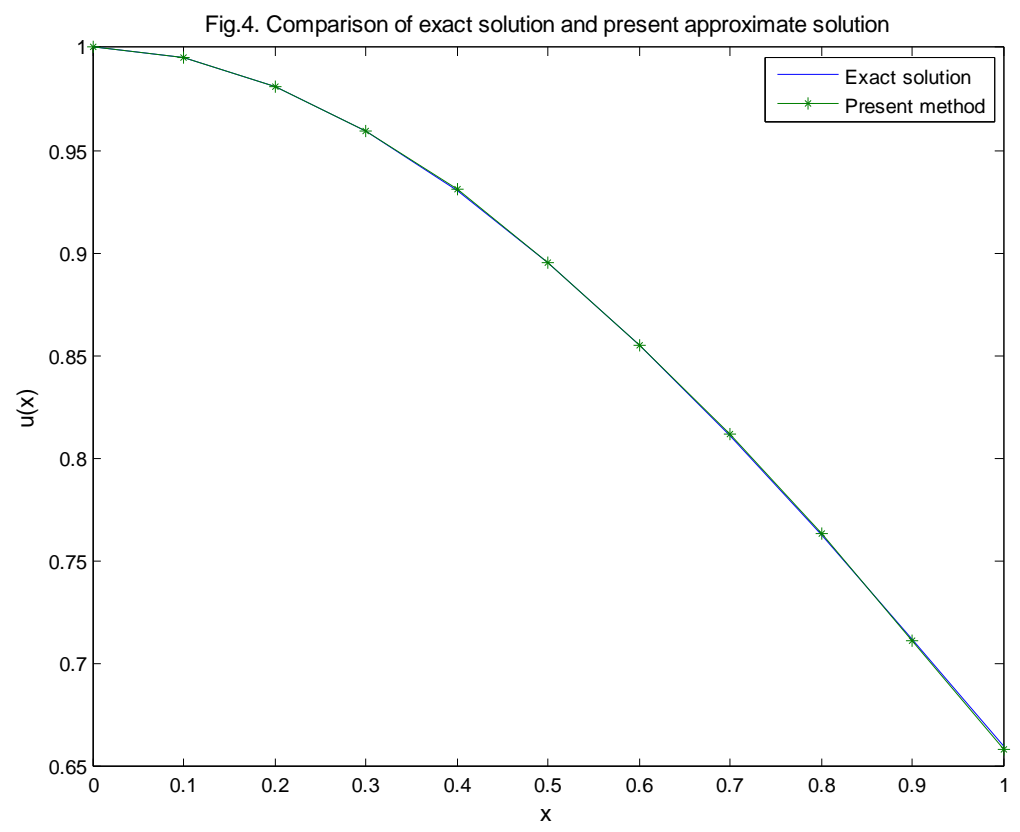

\section{CONCLUSION}

In this paper we have gone through a new insight into the use of Chebyshev polynomials. The series solutions in terms of Chebyshev polynomials have been used as numerically inverting Laplace transform tool for finding solutions of Volterra integral and integro-differential equations. The outcome of four test problems have been compared with exact or Pade approximants and found to be numerically efficient.

\section{REFERENCES}

[1] Bellman, R.E., H.H. Kagiwada and R.E. Kalba, Numerical Inversion of Laplace Transforms and Some Inverse Problems in Radiative Transfer, Journal of Atmospheric Sciences 23(1966), 555-559.

[2] Cohen, A.M., Numerical Methods for Laplace Transform Inversion, Springer, 2007.

[3] Dubner, H. and J. Abate, Numerical Inversion of Laplace Transforms by Relating them to the Finite Fourier Cosine Transform, Jour. Assoc. Comput. Math. 15 (1968), 115-123.

[4] Durbin, F., Numerical Inversion of Laplace Transforms: An efficient improvement to Dubner and Abate's method, Comput. Jour. 17 (1974), 371-376. 


\section{American Research Journal of Mathematics, Volume 1, Issue 1, February 2015}

ISSN 2378-704X

[5] Davies, Brian and Brian Martin, Numerical Inversion of Laplace Transform: A Survey and Comparison of Methods, Journal of Computational Physics 33(1979), 1-32.

[6] Papoulis, A., A New Method of Inversion of the Laplace Transform, Quart. Appl. Math. 14(1956), 405-414.

[7] Babolian, E. and A. Salimi Shamloo, Numerical Solution of Volterra Integral and Integro-differential Equations of Convolution Type by using Operational Matrices of Piecewise Constant Orthogonal Functions, Journal of Computational and Applied Mathematics 214 (2008), 495-508.

[8] Filiz, A. , Numerical Method for Linear Volterra Integro-differential Equation with Cash-karp Method, Asian Journal of Fuzzy and Applied Modeling 2 (2014), 1-11.

[9] Mishra, Vinod, Harpreet Kaur and R.C. Mittal, Haar Wavelet Solutions of Certain Differential, Integral and Integrodifferential Equations, International Journal of Applied Mathematics and Mechanics 8 (2012), 69-82.

[10] Raftari, B., Numerical Solution of Linear Volterra Integro-differential Equations: Homotopy Perturbation Method and Finite Difference Method, World Applied Sciences Journal 9 (2010), 7-12.

[11] Rashidinia, J. and Ali Tahmasebi, Approximate Solution of Linear Integro-differential Equation using Modified TaylorExpansion Method, World Journal of Modeling and Simulation 9 (2013), 289-301.

[12] Wazwaz, A.M., Linear and Nonlinear Integral Equations: Methods and Applications, Springer, 2011.

[13] Yang, Changqing and Jianhua Hou, Numerical Method for Solving Volterra Integral Equations with a Convolution Kernel, IAENG International Journal of Applied Mathematics 43 (2013), 1-5.

[14] Zarebnia, M. and Z. Nikpour, Solution of linear Volterra Integro-differential Equation via Sinc Functions, International Journal of Applied Mathematics and Computation 2 (2010), 1-10.

[15] Piessens, Robert, Computing Integral Transforms and Solving Integral Equations using Chebyshev Polynomial Approximations, Journal of Computational and Applied Mathematics 121 (2000) 113-124.

[16] Mishra Vinod, Review of Numerical Inversion of Laplace Transforms using Fourier Analysis, Fast Fourier Transform and Orthogonal Polynomials, Mathematics in Engineering, Science and Aerospace (USA) 5(2014), 239-261.

[17] Aznam, Suazlan Mt and Amran Hussin, Numerical Method for Inverse Laplace Transform with Haar Wavelet Operational Matrix, Malaysian Journal of Fundamental \& Applied Sciences 8 (2012), 182-188.

[18] Al-Hayani, Waleed, Solving nth-Order Integro-Differential Equations Using the Combined Laplace Transform-Adomian Decomposition Method, Applied Mathematics, 2013, 4, 882-886. 